Bernard L. G. Bakker • Chueng-Ryong Ji

\title{
The Construction of Compton Tensors in Scalar QED
}

Received: 31 August 2016 / Accepted: 2 November 2016 / Published online: 9 December 2016

(C) The Author(s) 2016. This article is published with open access at Springerlink.com

\begin{abstract}
Current conservation is a vital condition in electrodynamics. We review the literature concerning the ways to ensure that the formalism used in calculating amplitudes for the scattering of charged particles is in compliance with current conservation. For the case of electron scattering off a scalar and a spin-1/2 target as well as Compton scattering on a scalar target, we present some novelties besides reviewing the literature.
\end{abstract}

\section{Introduction}

The conservation of the electric current is a corner stone of electrodynamics, both classical and quantized. Textbooks like Refs. [1] and [2], to mention only two out of a plethora of references, write down the continuity equation

$$
\partial_{\mu} J^{\mu}=0
$$

which in the case of classical electrodynamics expresses the fact that in any volume the time derivative of the charge inside this volume is opposite to the divergence of the current flowing out through the surface. Noether's theorem states that in general such relations as Eq. (1) point to a conserved quantity, which of course in electrodynamics is the total charge.

In this paper we review some aspects of the construction of tensorial operators like the current $J^{\mu}$ in QED, which lead in the case of interacting theories to the concept of form factors. Naturally, once this concept is introduced, the question arises what is the number of independent form factors in each particular case. We shall not answer this question in general, but rather illustrate some methods used in three comparatively simple cases, namely the current of a charged particle in scalar QED (sQED) and spinor QED, and the construction of the Compton tensor in sQED.

While most of this paper is concerned with the existing literature, we add some novel results, that may help to gain a new perspective on the standard approaches.

This article belongs to the special issue "30th anniversary of Few-Body Systems".

B. L. G. Bakker ( $\square)$

Department of Physics and Astrophysics, Vrije Universiteit, Amsterdam, The Netherlands

E-mail: b.l.g.bakker@vu.nl

C.-R. Ji

Department of Physics, North Carolina State University, Raleigh, NC 27695-8202, USA

E-mail: crji@ncsu.edu 


\section{Electron Scattering Off a Scalar Target}

We start our discussion with the electron scattering off a scalar target to introduce the idea of parametrising the amplitude in terms of a tensor built with the help of the relevant momenta. In the case of a scalar target, three momenta are involved, the momenta $p$ and $p^{\prime}$ of the target before and after absorption of a virtual photon, and the momentum $q$ of the latter. Because of four-momentum conservation, $p+q=p^{\prime}$, only two out of three are independent. Dropping in this paper everywhere the magnitude of the charge of the target, which can easily be inserted in the photon-hadron vertices, we write the current operator as follows:

$$
J^{\mu}=F_{1} p^{\mu}+F_{2} p^{\prime \mu}
$$

Implementing the transversality condition Eq. (1), we find, using $q=p^{\prime}-p$

$$
0=q_{\mu} J^{\mu}=F_{1} p \cdot\left(p^{\prime}-p\right)+F_{2} p^{\prime} \cdot\left(p^{\prime}-p\right) .
$$

Using the on-mass-shell condition $p^{\prime 2}=p^{2}=M^{2}$ we find the relation $\left(F_{1}-F_{2}\right)\left(p^{\prime} \cdot p-M^{2}\right)=\left(F_{1}-\right.$ $\left.F_{2}\right) q^{2} / 2=0$. Because the photon can only be real, i. e. $q^{2}=0$, for the special kinematics where $p^{\prime}=p$, we see that $F_{1}=F_{2}$ almost everywhere in the kinematic space. The kinematical space for this scattering process is the three-dimensional space of independent dot products $p^{\prime 2}, p^{2}$, and $p^{\prime} \cdot p$.

Thus the final form of the electromagnetic current of a scalar particle can be written as

$$
J^{\mu}=F_{\mathrm{S}}\left(p^{\prime \mu}+p^{\mu}\right) \equiv F_{\mathrm{S}} \bar{P}^{\mu}
$$

We may call this the manifestly transverse form of the current, because the transversality condition is immediately seen to be satisfied by the momentum structure: $q \cdot \bar{P}=\left(p^{\prime}-p\right) \cdot\left(p^{\prime}+p\right)=p^{\prime 2}-p^{2}=M^{2}-M^{2}=0$. The same purpose is achieved if one writes the current in terms of a projector, namely in the form

$$
J^{\mu}=\left(g^{\mu \nu}-\frac{q^{\mu} q^{v}}{q^{2}}\right)\left(F_{\mathrm{S}} \bar{P}_{\nu}+F_{\mathrm{A}} q_{v}\right) \equiv \mathcal{G}^{\mu \nu}(q q)\left(F_{\mathrm{S}} \bar{P}_{\nu}+F_{\mathrm{A}} q_{\nu}\right)
$$

The projector $\mathcal{G}^{\mu \nu}(q q)$ annihilates $q^{\mu}$ and $q^{\nu}$, which property guarantees the transversality of the current and removes the form factor $F_{\mathrm{A}}$. Thus an equivalent form of the current operator would be

$$
J^{\mu}=F_{\mathrm{S}} \mathcal{G}^{\mu \nu}(q q) \bar{P}_{\nu}=F_{\mathrm{S}}\left(\bar{P}^{\mu}-\frac{\bar{P} \cdot q}{q^{2}} q^{\mu}\right) .
$$

The two forms, Eqs. (4) and (6), are equivalent in the part of the kinematical space where $q^{2} \neq 0$, but not in the singular subspace where $q^{2}$ vanishes. Such situations will be seen in the Compton-scattering case too. Let us note that although the vector part may vary, the number of form factors (FFs) is invariant.

If the target is not structureless, but can be excited, the argument for a single form factor does not apply anymore: there must exist an elastic form factor plus transition form factors. For the purpose of this paper we shall only consider the situation where $p^{\prime}$ and $p$ are on the same mass shell.

We shall later use the same notation, $\bar{P}=p^{\prime}+p$, when we discuss Compton scattering.

\section{Electron Scattering Off a Spinor Target}

Was the form of the electromagnetic current of a scalar particle almost unique, for a spin-1/2 particle it is not. In fact, one can write it in different forms. The text books usually give two forms of the current, namely one with the Dirac and Pauli FFs [3]

$$
J^{\mu}=\gamma^{\mu} F_{1}+i \frac{\sigma^{\mu v} q_{v}}{2 M} F_{2} .
$$

Here the normalisation of $F_{2}$ is such that $F_{2}\left(q^{2}=0\right)$ is equal to the anomalous magnetic moment.

Ernst et al. [4] introduced a redefinition of the FFs, namely

$$
G_{\mathrm{E}}=F_{1}+\frac{q^{2}}{4 M^{2}} F_{2}, \quad G_{\mathrm{M}}=F_{1}+F_{2},
$$


where $G_{\mathrm{E}}$ and $G_{\mathrm{M}}$ are the electric and magnetic FFs [5]. Such a redefinition is equivalent to reshuffling the operators in the expression for the current. In the derivation of the current operator, Bjorken and Drell [3] allow for three terms, namely $F_{1} p^{\prime \mu}+F_{2} p^{\mu}+F_{3} \gamma^{\mu}$ and show that using the on-shellness of the momenta $p^{\prime}$ and $p$ and current conservation, that the operator could be reduced to $F_{1}\left(p^{\prime \mu}+p^{\mu}\right)+F_{3} \gamma^{\mu}$. Now a key ingredient is the Gordon decomposition [6]:

$$
\bar{u}\left(p^{\prime}\right) \gamma^{\mu} u(p)=\frac{1}{2 M} \bar{u}\left(p^{\prime}\right)\left(\left(p^{\prime}+p\right)^{\mu}+i \sigma^{\mu \nu} q_{v}\right) u(p) .
$$

Besides this classic form, one may also get the following decomposition [7]:

$$
\bar{u}\left(p^{\prime}\right) i \sigma^{\mu \nu} q_{\nu} u(p)=\frac{1}{2 M} \bar{u}\left(p^{\prime}\right)\left(q^{2} \gamma^{\mu}+2 i \epsilon^{\mu \nu \alpha \beta} \gamma_{5} \gamma_{\nu} p_{\alpha}^{\prime} p_{\beta}\right) u(p) .
$$

Using both decompositions we altogether arrive at six different forms of the spin-1/2 electromagnetic current operator:

$$
\begin{aligned}
J^{\mu} & =\gamma^{\mu} F_{1}+i \frac{\sigma^{\mu \nu} q_{v}}{2 M} F_{2}, \\
& =\gamma^{\mu}\left(F_{1}+F_{2}\right)-\frac{\left(p^{\prime}+p\right)^{\mu}}{2 M} F_{2}, \\
& =\frac{\left(p^{\prime}+p\right)^{\mu}}{2 M} \frac{4 M^{2} F_{1}+q^{2} F_{2}}{4 M^{2}-q^{2}}+i \epsilon^{\mu \nu \alpha \beta} \gamma_{5} \gamma_{\nu} p_{\alpha}^{\prime} p_{\beta} \frac{2\left(F_{1}+F_{2}\right)}{4 M^{2}-q^{2}}, \\
& =\frac{\left(p^{\prime}+p\right)^{\mu}}{2 M} F_{1}+i \frac{\sigma^{\mu \nu} q_{v}}{2 M} i\left(F_{1}+F_{2}\right), \\
& =\gamma^{\mu}\left(F_{1}+\frac{q^{2}}{4 M^{2}} F_{2}\right)+i \epsilon^{\mu \nu \alpha \beta} \gamma_{5} \gamma_{\nu} p_{\alpha}^{\prime} p_{\beta} \frac{F_{2}}{2 M^{2}}, \\
& =i \frac{\sigma^{\mu \nu} q_{v}}{2 M}\left(\frac{4 M^{2}}{q^{2}} F_{1}+F_{2}\right)-i \epsilon^{\mu \nu \alpha \beta} \gamma_{5} \gamma_{\nu} p_{\alpha}^{\prime} p_{\beta} \frac{2 F_{1}}{q^{2}} .
\end{aligned}
$$

Several remarks must be made. First, these forms are not identical, but give identical matrix elements between spinors, which in operator theory is defined as weak identity. Secondly, neither of the two decompositions Eqs. (9) and (10) can be derived for massless spinors, as the occurrences of an $M$ in the denominators in these equations show. Thirdly, some of these forms are manifestly transverse to $q_{\mu}$, namely the third, fourth

and sixth one. Transversality is trivially proven by the identity we used before, $\bar{P} \cdot q=0$, and taking into account that $\sigma^{\mu \nu}$ as well as $\epsilon^{\mu \nu \alpha \beta}$ are antisymmetric in all indices.

\section{Compton Scattering Off a Scalar Target}

Real Compton scattering has been an interesting phenomenon since its interpretation by Compton [8] as well as Debye [9] played an important role in the reception of the light-quanta hypothesis [10] before quantum mechanics was established. Presently, virtual Compton scattering $[11,12]$ has been proposed to determine the generalized-parton distributions (GPDs) of hadrons. In this interpretation, the handbag diagram, as shown in Fig. 1, plays a crucial role. In a more general setting, one considers the hadronic part of the amplitude, which

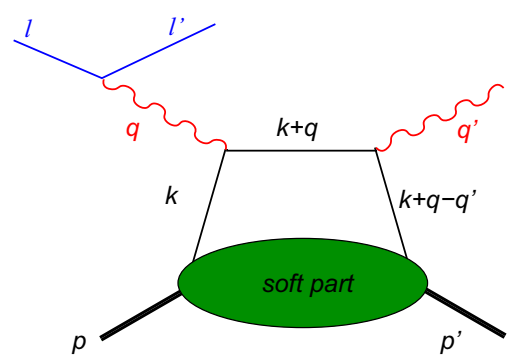

Fig. 1 Handbag diagram, including the leptonic part 
describes the absorption of a virtual photon and the emission of a real photon by a hadron, without committing oneself from the beginning to the structure of the hadron except for its mass and spin, remaining oblivious to its (quark) constituents.

In this paper we shall remain on that level, discussing only the number of independent scalar functions, Compton form factors (CFFs) that can be distinguished, like the form factors in the case of the vector currents discussed in the previous sections.

\subsection{Tensor Formalism}

In (virtual) Compton scattering the physical invariant amplitudes are written as contractions of a tensor with the polarization vectors of the photons:

$$
A\left(q^{\prime}, h^{\prime} ; q, h\right)=\epsilon_{\mu}^{*}\left(q^{\prime} ; h^{\prime}\right) T^{\mu \nu} \epsilon_{\nu}(q ; h) .
$$

The tensor $T^{\mu v}$ must satisfy two transversality conditions, which are related to current conservation or gauge invariance, namely

$$
q_{\mu}^{\prime} T^{\mu \nu}=0, \quad T^{\mu v} q_{v}=0 .
$$

One may connect these relations also to the invariance of the amplitudes $A\left(q^{\prime}, h^{\prime} ; q, h\right)$ under Lorentz transformations. As discussed in Ref. [2], Ch. 8, the vector potential $A^{\mu}$ in QED transforms under a Lorentz transformation as a four vector only up to a gauge transformation. In the present context it means that under a Lorentz transformation the polarisation vector $\epsilon^{\mu}(q ; h)$ changes into the formally Lorentz transformed vector $\Lambda_{\nu}^{\mu} \epsilon^{v}(q ; h)$ plus a multiple of the momentum vector $q^{\mu}$. Thus the invariance of the amplitude is guaranteed by the transversality of the Compton tensor.

Two problems now present themselves: how to determine the number of effective degrees of freedom, i.e. the number of CFFs, and how to construct a tensor that satisfies the transversality conditions Eq. (13)? We shall sketch several ways to solve these two problems. The first one, which to the best of our knowledge was not mentioned in the physics literature, is based on a straightforward application of linear algebra. A number of constructions has been given before and we shall review the ones that are essentially different.

As a preliminary we now define our notation. To start with, we use $q\left(q^{\prime}\right)$ for the absorbed (emitted) photons and $p\left(p^{\prime}\right)$ for the target (recoiled) hadron. Because of four-momentum conservation, three out of these four momenta are independent. We shall choose the following three:

$$
k_{1}=\bar{P}=p^{\prime}+p, \quad k_{2}=q^{\prime}, \quad k_{3}=q .
$$

For coherent Compton scattering, where the recoiled hadron is identical to the target, one easily derives the following identities

$$
\begin{aligned}
2 \bar{P} \cdot q & =2 \bar{P} \cdot q^{\prime}=s-M^{2}-\left(u-M^{2}\right), \\
(\bar{P}+q) \cdot q^{\prime} & =\left(\bar{P}+q^{\prime}\right) \cdot q=s-M^{2}, \\
(\bar{P}-q) \cdot q^{\prime} & =\left(\bar{P}-q^{\prime}\right) \cdot q=-\left(u-M^{2}\right), \\
2 q^{\prime} \cdot q & =s-M^{2}+u-M^{2},
\end{aligned}
$$

where $s$ and $u$ are the usual Mandelstam variables $s=(p+q)^{2}=\left(p^{\prime}+q^{\prime}\right)^{2}$ and $u=\left(p-q^{\prime}\right)^{2}=\left(p^{\prime}-q\right)^{2}$.

Using the notation $k_{i}, i=1,2,3$ for the momenta, we can write for the most general tensor of rank two in the following form

$$
T^{\mu \nu}=t_{0} g^{\mu \nu}+\sum_{i=1}^{3} \sum_{j=1}^{3} t_{i j} K_{i j}^{\mu \nu}
$$

with

$$
K_{i j}^{\mu \nu}=k_{i}^{\mu} k_{j}^{\nu}
$$

The ten quantities $t_{0}, t_{11}, \ldots t_{33}$ are scalar quantities. If no conditions are set for the tensor $T^{\mu \nu}$, we see that it has ten degrees of freedom. Thus, instead of the representation given in Eq. (16) we could, interpreting the set $g^{\mu \nu}, K_{11}^{\mu \nu}, \ldots, K_{33}^{\mu \nu}$ as a basis in a 10-dimensional linear space, interpret the tensor as the contraction of the vector $t$ with scalar elements $t_{0}, t_{11}, \ldots, t_{33}$ with the vector of basis tensors $g^{\mu \nu}, K_{11}^{\mu \nu}, \ldots, K_{33}^{\mu \nu}$. This interpretation lends itself immediately to the application of the theory of vector spaces, which is the content of what we call the direct method. 


\subsubsection{Direct Method}

Understanding Eq. (16) as expressing the full tensor as a linear combination of basis tensors leads to the treatment of the transversality conditions as conditions on the vector $t$. Let us write the contraction $q^{\prime}{ }_{\mu} T^{\mu \nu}$ as follows

$$
k_{2 \mu} T^{\mu \nu}=t_{0} k_{2}^{\nu}+\sum_{i, j} t_{i j}\left(k_{2} \cdot k_{i}\right) k_{j}^{\nu}
$$

Similarly, we write the other contraction as

$$
T^{\mu v} k_{3 v}=t_{0} k_{3}^{\mu}+\sum_{i, j} t_{i j}\left(k_{3} \cdot k_{j}\right) k_{i}^{\mu} .
$$

The condition that these two contractions vanish, leads to two sets of three linear equations in the components of $t$. We shall use the notation

$$
x_{i j}=k_{i} \cdot k_{j} .
$$

From Eq. (15) we read off that $\bar{P} \cdot q^{\prime}=x_{12}=x_{13}=\bar{P} \cdot q$. Taking into account that the basis vectors $k_{i}$ are independent four vectors, we find the equations in the following form

$$
X \cdot t=0
$$

where the matrix $X$ is given by

$$
X=\left(\begin{array}{cccccccccc}
0 & x_{13} & 0 & 0 & x_{22} & 0 & 0 & x_{23} & 0 & 0 \\
1 & 0 & x_{13} & 0 & 0 & 0 & x_{22} & 0 & x_{23} & 0 \\
0 & 0 & 0 & x_{13} & 0 & 0 & x_{22} & 0 & 0 & x_{23} \\
0 & x_{13} & x_{23} & x_{33} & 0 & 0 & 0 & 0 & 0 & 0 \\
0 & 0 & 0 & & x_{13} & x_{23} & x_{23} & 0 & 0 & 0 \\
1 & 0 & 0 & 0 & 0 & 0 & 0 & x_{13} & x_{23} & x_{33}
\end{array}\right) .
$$

Now the two problems can be formulated as follows: what is the matrix rank of $X$ and what is the null-space of this matrix? The answer to the first question gives the number of independent scalars, i.e. the number of $\mathrm{CFFs}$, and the answer to the second question gives a way to write down the general form of a rank-two tensor $\tilde{T}^{\mu v}$ that is transverse to $q^{\prime}$ (left) and $q$ (right).

The first question can be answered in two ways. The first one is a straightforward application of Gaussian elimination to find that the matrix rank of $X$ is five. Indeed one finds that the following linear combination of the rows of $X$ vanishes

$$
\bar{P} \cdot q \operatorname{row}_{1}+q^{\prime} \cdot q \text { row }_{2}+q^{2} \text { row }_{3}-\bar{P} \cdot q \text { row }_{4}-q^{\prime 2} \text { row }_{5}-q^{\prime} \cdot q \text { row }_{6}=0
$$

The second one is to contract $T^{\mu \nu}$ simultaneously with $k_{i \mu}$ and $k_{j v}$ and to find six contractions to vanish, namely the ones involving $k_{2 \mu}$ or $k_{3 \nu}$. In this set the double contraction with $k_{2 \mu}$ and $k_{3 \nu}$ occurs twice, leaving at most five independent ones.

Now that we know that only five scalars are involved in a general tensor that is transverse to $q_{\mu}^{\prime}$ and $q_{\nu}$, we can write such a tensor as a linear combination of a basis in the five-dimensional null-space $\mathcal{N}_{X}$ of the matrix $X$. Let us note here that while the matrix $X$ is uniquely defined and thus so is its null-space, one may use any basis one likes in $\mathcal{N}_{X}$. This is the reason why there exist in the literature several forms of the Compton tensor.

A final point to consider is the issue of kinematical singularities. The coefficients in the linear combination Eq. (23) may vanish. At this point the most interesting ones are $q^{\prime 2}=0$ and $q^{2}=0$. If both conditions apply, we are dealing with real Compton scattering (RCS), otherwise the tensor $T^{\mu v}$ is used to calculate the amplitudes for virtual Compton scattering (VCS) or even electro-production of vector states. Calculating the matrix rank of $X$ one finds that only either in the case that all coefficients in Eq. (23) vanish or in the case that all but $q^{2}$ or $q^{\prime 2}$ vanish, the ranks is lowered to either 1 or 4 , respectively. Otherwise it remains five. However, we note that the simultaneous vanishing of all scalar products $x_{i j}$ is kinematically forbidden. To see why, we write them down and calculate them explicitly:

$$
\begin{aligned}
& x_{13}=\bar{P} \cdot q^{\prime}=\bar{P} \cdot q=0, \\
& x_{22}=q^{\prime 2}=0, \\
& x_{23}=q^{\prime} \cdot q=0, \\
& x_{33}=q^{2}=0 .
\end{aligned}
$$



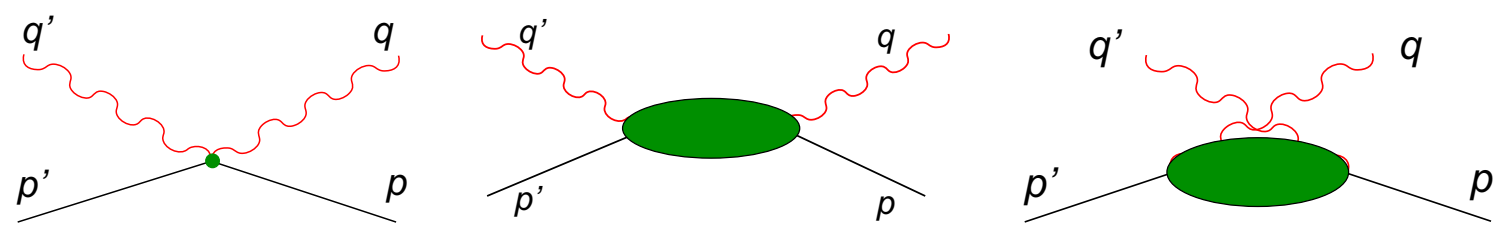

Fig. 2 Seagull, $s$ - and $u$-channel amplitudes

We may thus write $q^{\prime \mu}=q^{\prime}\left(1, \hat{q}^{\prime}\right), q^{\mu}=q(1, \hat{q})$ and find that $x_{23}=0$ implies that $\hat{q}^{\prime}=\hat{q}$. Let us write $\bar{P}^{\mu}=\left(P^{0}, \boldsymbol{P}\right)$. For $x_{13}$ to vanish two conditions must be satisfied: (i) $\boldsymbol{P}$ is parallel to $\boldsymbol{q}^{\prime}$ and (ii) $P^{0}$ must be equal to the length of $\boldsymbol{P}$. While the first condition is kinematically possible, the latter is not, because it means that $\bar{P}$ is light-like, which it is not. Substituting $p^{\prime 2}=p^{2}=M^{2}$ and using $t=\left(p^{\prime}-p\right)^{2}=2 M^{2}-2 p^{\prime} \cdot p=$ $\left(q-q^{\prime}\right)^{2}=q^{\prime 2}+q^{2}-2 q^{\prime} \cdot q$ we find

$$
\bar{P}^{2}=\left(p^{\prime}+p\right)^{2}=2 M^{2}+2 p^{\prime} \cdot p=4 M^{2}-t=4 M^{2},
$$

where we have used $x_{22}=x_{23}=x_{33}=0$. Thus because $\bar{P}$ cannot be light-like, the matrix $X$ has for all physical situations and for finite target mass $M$ matrix rank five.

Although we are now assured that the number of CFFs is five, it does not mean that the null-spaces of $X$ in the case that one or several dot products $x_{i j}$ vanish must be identical. For illustrative reasons we show a basis of the null-space for the general case, substituting the dot products of physical momenta:

$$
\left(\begin{array}{l}
n^{(1)} \\
n^{(2)} \\
n^{(3)} \\
n^{(4)} \\
n^{(5)}
\end{array}\right)=\left(\begin{array}{cccccccccc}
-q^{\prime 2} & 0 & \frac{q^{2}}{\bar{P} \cdot q} & -\frac{q^{\prime} \cdot q}{\bar{P} \cdot q} & 0 & 0 & 0 & 0 & 0 & 1 \\
-q^{\prime} \cdot q & 0 & 0 & 0 & 0 & 0 & 0 & 0 & 1 & 0 \\
-\bar{P} \cdot q & -\frac{q^{\prime} \cdot q}{\bar{P} \cdot q} & 1 & 0 & 0 & 0 & 0 & 1 & 0 & 0 \\
0 & \frac{q^{2} q^{\prime 2}}{\bar{P} \cdot q)^{2}} & 0 & -\frac{q^{\prime 2}}{\bar{P} \cdot q} & -\frac{q^{2}}{\bar{P} \cdot q} & 0 & 1 & 0 & 0 & 0 \\
0 & \frac{q^{\prime} \cdot q q^{\prime 2}}{(\bar{P} \cdot q)^{2}} & -\frac{q^{2}}{\bar{P} \cdot q} & 0 & -\frac{q^{\prime} \cdot q}{\bar{P} \cdot q} & 1 & 0 & 0 & 0 & 0
\end{array}\right) .
$$

Clearly, this basis of $\mathcal{N}_{X}$ demonstrates that the value $\bar{P} \cdot q=0$ is a singular point in the null-space of $X$. It would not help to multiply the basis vectors with a power of $\bar{P} \cdot q$ to remove the singularity, because the resulting basis, while still independent for $\bar{P} \cdot q \neq 0$, would become incomplete. In the latter situation one can determine a new basis for $\mathcal{N}_{X}$, that would again be five-dimensional. However, this time the basis will become singular if $q^{\prime 2}=0$ or $q^{\prime} \cdot q$ would vanish. This is an illustration of a conclusion already formulated in a CERN report by Callan and Stora [11,12], that it is not possible to construct a basis for $\mathcal{N}_{X}$ that spans the whole null-space for all physical values of the dot products $x_{i j}$ and is free of kinematic singularities. We shall see that the other methods will meet the same problem.

Finally we illustrate the construction of the Compton tensor described in this section by writing it for the tree-level case and show how it is expanded in the given basis. We shall use this example to illustrate also the constructions reviewed below. The tree level $T^{\mu \nu}$ is given by

$$
T_{\text {tree }}^{\mu \nu}=-2 g^{\mu \nu}+\frac{(\bar{P}+q)^{\mu}\left(\bar{P}+q^{\prime}\right)^{v}}{s-M^{2}}+\frac{(\bar{P}-q)^{\mu}\left(\bar{P}-q^{\prime}\right)^{v}}{u-M^{2}} .
$$

This tensor satisfies the transversality conditions, as it must of course. To facilitate the demonstration that $T_{\text {tree }}^{\mu \nu}$ can be expanded in the basis we found before, Eq. (26), we write the denominators in terms of the dot products of basis four vectors using Eq. (15): $s-M^{2}=x_{13}+x_{23}$, and $u-M^{2}=x_{13}-x_{23}$. The result for the $t$-vector corresponding to $T_{\text {tree }}^{\mu \nu}$ is

$$
t_{\text {tree }}=-\frac{2}{(\bar{P} \cdot q)^{2}-\left(q^{\prime} \cdot q\right)^{2}}\left[-q^{\prime} \cdot q n^{(2)}+\bar{P} \cdot q n^{(3)}\right]
$$


where we have substituted the physical values for the dot products. Thus the tree-level CFFs in this formulation are given by

$$
\mathcal{C}_{1}=-2 \frac{q^{\prime} \cdot q}{(\bar{P} \cdot q)^{2}-\left(q^{\prime} \cdot q\right)^{2}}=\frac{1}{s-M^{2}}+\frac{1}{u-M^{2}}, \quad \mathcal{C}_{2}=2 \frac{\bar{P} \cdot q}{(\bar{P} \cdot q)^{2}-\left(q^{\prime} \cdot q\right)^{2}}=\frac{1}{s-M^{2}}-\frac{1}{u-M^{2}} .
$$

The fact that in $T_{\text {tree }}^{\mu v}$ only two CFFs occur is not due to kinematical singularities but to the limited dynamics involved in a tree-level amplitude.

\subsubsection{Projection Methods}

The relative opacity of the direct method may be the reason that one does not find it discussed in the literature. Instead, one can find several treatments of expansions of the Compton tensor as a sum of transverse basis tensors. We shall briefly discuss two proposals, one by Perrottet [13], the other by Tarrach [14] with the explicit application to the sQED case by Metz [15]. These authors treat the situation where the target hadron is a proton, but their methods include the case where the target has spin zero.

Perrottet uses the idea, presented already above for the single-photon case, to use projection operators to construct the transversal Compton tensor. In particular, he uses the projectors $\mathcal{P}$ and $\mathcal{P}^{\prime}$ given by

$$
\mathcal{P}^{\mu \nu}=g^{\mu \nu}-\frac{q^{\mu} q^{\nu}}{q^{2}}, \quad \mathcal{P}^{\prime \mu \nu}=g^{\mu \nu}-\frac{q^{\prime \mu} q^{\prime \nu}}{q^{\prime 2}} .
$$

Because these projectors annihilate $q$ and $q^{\prime}$, respectively, they can be used to turn a general tensor $T^{\mu \nu}$ into a transversal one:

$$
\tilde{T}^{\mu \nu}=\mathcal{P}^{\prime \mu m} T_{m n} \mathcal{P}^{n v}
$$

Because the projectors that are used by Perrottet are singular for real photons, this method has a limited domain of application. An improvement was achieved by Tarrach, who constructs the transversal tensor $T^{\mu \nu}$ by applying a two-sided projector $\tilde{g}^{\mu \nu}\left(q, q^{\prime}\right)$, given by

$$
\tilde{g}^{\mu \nu}\left(q, q^{\prime}\right)=g^{\mu \nu}-\frac{q^{\mu} q^{\prime \nu}}{q \cdot q^{\prime}}
$$

to $T^{\mu \nu}$ :

$$
\tilde{T}^{\mu \nu}=\tilde{g}^{\mu m} T_{m n} \tilde{g}^{n v} .
$$

The projector $\tilde{g}^{\mu \nu}\left(q, q^{\prime}\right)$ is idempotent, i.e., its contraction with itself is equal to $\tilde{g}^{\mu \nu}\left(q, q^{\prime}\right)$ again.

Because $\tilde{g}^{\mu \nu}\left(q, q^{\prime}\right)$ contracted from the left with $T^{\mu \nu}$ removes the terms with $q^{\mu} k_{j}^{v}$ and contracted from the right it does the same with terms of the form $k_{i}^{\mu} q^{\prime \nu}$, the result of applying $\tilde{g}^{\mu \nu}\left(q, q^{\prime}\right)$ simultaneously from the left and from the right to $T^{\mu \nu}$ immediately produces a transversal tensor with only five independent terms. Defining the reduced momenta, $\left(k=\bar{P}, q^{\prime}, q\right)$ :

$$
\tilde{k}_{\mathrm{L}}^{\mu}=\tilde{g}^{\mu v} k_{v}, \quad \tilde{k}_{\mathrm{R}}^{v}=k_{\mu} \tilde{g}^{\mu v}
$$

one finds the following result for $\tilde{T}^{\mu \nu}$

$$
\tilde{T}^{\mu \nu}=\mathcal{H}_{0} \tilde{g}^{\mu \nu}+\mathcal{H}_{1} \tilde{P}_{\mathrm{L}}^{\mu} \tilde{P}_{\mathrm{R}}^{v}+\mathcal{H}_{2} \tilde{P}_{\mathrm{L}}^{\mu} \tilde{q}_{\mathrm{R}}^{\nu}+\mathcal{H}_{3} \tilde{q}_{\mathrm{L}}^{\prime \mu} \tilde{P}_{\mathrm{R}}^{v}+\mathcal{H}_{4} \tilde{q}_{\mathrm{L}}^{\prime \mu} \tilde{q}_{\mathrm{R}}^{v}:=\sum_{a=0}^{4} \mathcal{H}_{a} \tilde{T}_{a}^{\mu \nu}
$$

While we saw previously that kinematical singularities arise using the null-space basis, we see now that they also arise using the method of projectors. The difference with the one we saw explicitly in the previous subsection, namely the point $\bar{P} \cdot q=0$, is that now the singularity is at $q^{\prime} \cdot q=0$. If we should have used 
another basis for the null-space $\mathcal{N}_{X}$ we could have removed the singularity at $\bar{P} \cdot q=0$ but would find another one, perhaps two as in the Perrottet construction. The null-space basis corresponding to Eq. (35) is

$$
\left(\begin{array}{l}
n_{T}^{(1)} \\
n_{T}^{(2)} \\
n_{T}^{(3)} \\
n_{T}^{(4)} \\
n_{T}^{(5)}
\end{array}\right)=\left(\begin{array}{cccccccccc}
1 & 0 & 0 & 0 & 0 & 0 & 0 & 0 & -\frac{1}{q^{\prime} \cdot q} & 0 \\
0 & 1 & -\frac{\bar{P} \cdot q}{q^{\prime} \cdot q} & 0 & 0 & 0 & 0 & -\frac{\bar{P} \cdot q}{q^{\prime} \cdot q} & \frac{(\bar{P} \cdot q)^{2}}{\left(q^{\prime} \cdot q\right)^{2}} & 0 \\
0 & 0 & -\frac{q^{2}}{q^{\prime} \cdot q} & 1 & 0 & 0 & 0 & 0 & \frac{q^{2} \bar{P} \cdot q}{\left(q^{\prime} \cdot q\right)^{2}} & -\frac{\bar{P} \cdot q}{q^{\prime} \cdot q} \\
0 & 0 & 0 & 0 & 1 & -\frac{\bar{P} \cdot q}{q^{\prime} \cdot q} & 0 & -\frac{q^{\prime 2}}{q^{\prime} \cdot q} & \frac{q^{\prime 2} \cdot \frac{\bar{P}}{P} \cdot q}{\left(q^{\prime} \cdot q\right)^{2}} & 0 \\
0 & 0 & 0 & 0 & 0 & -\frac{q^{2}}{q^{\prime} \cdot q} & 1 & 0 & \frac{q^{\prime 2} q^{2}}{\left(q^{\prime} \cdot q\right)^{2}} & -\frac{q^{\prime 2}}{q^{\prime} \cdot q} \cdot
\end{array}\right) .
$$

This basis shows explicitly that the singularity in the projection operator also occurs in the the null-space basis.

In Tarrach's paper a method is described to remove the kinematic poles, which is a generalisation of the method given by Bardeen and Tung [16] for real Compton scattering. It consists in first taking linear combinations of the basis tensors presented in Eq. (35) to eliminate as many poles as possible. Next, if necessary, multiply any remaining double poles by $q^{\prime} \cdot q$ and again take linear combinations to remove as many single poles as possible. If any single poles are left, the final step consists in multiplying them with $q^{\prime} \cdot q$.

The form of the transverse tensor given in Eq. (35) is regularised by first adding a multiple of $\tilde{T}_{0}$ which contains only a single pole, to the four tensors with both single and double poles, multiplied by $q^{\prime} \cdot q$. Finally $\tilde{T}_{0}$ is also multiplied by $q^{\prime} \cdot q$. The result for the regularised tensors denoted with the subscript $\mathrm{R}$, is

$$
\begin{aligned}
& \tilde{T}_{0 R}^{\mu \nu}=q^{\prime} \cdot q \tilde{T}_{0}^{\mu \nu}=q^{\prime} \cdot q g^{\mu \nu}-q^{\mu} q^{\prime \nu} \\
& \tilde{T}_{1 R}^{\mu \nu}=(\bar{P} \cdot q)^{2} \tilde{T}_{0}^{\mu \nu}+q^{\prime} \cdot q \tilde{T}_{1}^{\mu \nu}=(\bar{P} \cdot q)^{2} g^{\mu \nu}+q^{\prime} \cdot q \bar{P}^{\mu} \bar{P}^{\nu}-\bar{P} \cdot q\left(\bar{P}^{\mu} q^{\prime \nu}+q^{\mu} \bar{P}^{\nu}\right), \\
& \tilde{T}_{2 R}^{\mu \nu}=\bar{P} \cdot q q^{2} \tilde{T}_{0}^{\mu \nu}+q^{\prime} \cdot q \tilde{T}_{2}^{\mu \nu}=\bar{P} \cdot q q^{2} g^{\mu \nu}-q^{2} \bar{P}^{\mu} q^{\prime \nu}+q^{\prime} \cdot q \bar{P}^{\mu} q^{\nu}-\bar{P} \cdot q q^{\mu} q^{\nu}, \\
& \tilde{T}_{3 R}^{\mu \nu}=\bar{P} \cdot q q^{\prime 2} \tilde{T}_{0}^{\mu \nu}+q^{\prime} \cdot q \tilde{T}_{3}^{\mu \nu}=\bar{P} \cdot q q^{\prime 2} g^{\mu \nu}+q^{\prime} \cdot q q^{\prime \mu} \bar{P}^{\nu}-q^{\prime 2} q^{\mu} \bar{P}^{\nu}-\bar{P} \cdot q q^{\prime \mu} q^{\prime \nu}, \\
& \tilde{T}_{4 R}^{\mu \nu}=q^{\prime 2} q^{2} \tilde{T}_{0}^{\mu \nu}+q^{\prime} \cdot q \tilde{T}_{4}^{\mu \nu}=q^{\prime 2} q^{2} g^{\mu \nu}-q^{2} q^{\prime \mu} q^{\prime \nu}+q^{\prime} \cdot q q^{\prime \mu} q^{\nu}-q^{\prime 2} q^{\mu} q^{\nu} .
\end{aligned}
$$

When the limit $q^{\prime} \cdot q \rightarrow 0$ is taken, these regularised tensors are not complete, because we can write for instance $\tilde{T}_{4 R}^{\mu \nu}$ as a linear combination of three others, namely

$$
\lim _{q^{\prime} \cdot q \rightarrow 0} \tilde{T}_{4 R}^{\mu \nu}=-\frac{1}{\bar{P} \cdot q}\left[\frac{q^{\prime 2} q^{2}}{\bar{P} \cdot q} \tilde{T}_{1 R}^{\mu \nu}-q^{\prime 2} \tilde{T}_{2 R}^{\mu \nu}+q^{2} \tilde{T}_{3 R}^{\mu \nu}\right] .
$$

Because Metz uses in his thesis [15] a basis for $T^{\mu \nu}$ different from the one we use here, the final result obtained by him using Tarrach's algorithm differs from the result Eq. (37), namely

$$
\begin{aligned}
M_{1}^{\mu \nu}= & -q^{\prime} \cdot q g^{\mu \nu}+q^{\mu} q^{\prime \nu}, \\
M_{2}^{\mu \nu}= & -(\bar{P} \cdot q)^{2} g^{\mu \nu}-q^{\prime} \cdot q \bar{P}^{\mu} \bar{P}^{\nu}+\bar{P} \cdot q\left(\bar{P}^{\mu} q^{\prime \nu}+q^{\mu} \bar{P}^{\nu}\right), \\
M_{3}^{\mu \nu}= & q^{\prime 2} q^{2} g^{\mu \nu}+q^{\prime} \cdot q q^{\prime \mu} q^{\nu}-q^{2} q^{\prime \mu} q^{\prime \nu}-q^{\prime 2} q^{\mu} q^{\nu}, \\
M_{4}^{\mu \nu}= & \bar{P} \cdot q\left(q^{\prime 2}+q^{2}\right) g^{\mu \nu}-\bar{P} \cdot q\left(q^{\prime \mu} q^{\prime \nu}+q^{\mu} q^{\nu}\right) \\
& -q^{2} \bar{P}^{\mu} q^{\prime \nu}-q^{\prime 2} q^{\mu} \bar{P}^{\nu}+q^{\prime} \cdot q\left(\bar{P}^{\mu} q^{\nu}+q^{\prime \mu} \bar{P}^{\nu}\right), \\
M_{19}^{\mu \nu}= & (\bar{P} \cdot q)^{2} q^{\prime \mu} q^{\nu}+q^{\prime 2} q^{2} \bar{P}^{\mu} \bar{P}^{\nu}-\bar{P} \cdot q q^{2} q^{\prime \mu} \bar{P}^{\nu}-\bar{P} \cdot q q^{\prime 2} \bar{P}^{\mu} q^{\nu} .
\end{aligned}
$$

(For historical reasons, the fifth tensor has subscript 19).

As an example we give the tree-level amplitude. In Tarrach's basic formulation of the CFFs one finds:

$$
\mathcal{H}_{0}^{\text {tree }}=-2, \mathcal{H}_{1}^{\text {tree }}=\frac{1}{s-M^{2}}+\frac{1}{u-M^{2}}, \mathcal{H}_{2}^{\text {tree }}=0, \mathcal{H}_{3}^{\text {tree }}=0, \mathcal{H}_{4}^{\text {tree }}=0,
$$

where we use the convention of Eq. (35) for the CFFs. In Metz's formulation one finds, using the symbol $\mathcal{B}$ for the CFFs:

$$
\mathcal{B}_{1}^{\text {tree }}=\frac{1}{s-M^{2}}+\frac{1}{u-M^{2}}, \mathcal{B}_{2}^{\text {tree }}=-\frac{2}{\left(s-M^{2}\right)\left(u-M^{2}\right)}, \mathcal{B}_{3}^{\text {tree }}=0, \mathcal{B}_{4}^{\text {tree }}=0, \mathcal{B}_{19}^{\text {tree }}=0 .
$$


where we use the numbering in Eq. (39).

We have found three different results for the form of the Compton tensor, even in the simplest case, namely tree-level. This demonstrates that the choice of the basis elements used in $T^{\mu \nu}$, apart from the fact that there are only three independent four vectors to choose from, matters in identifying the CFFs. In general, one will find linear relations between the tensors used in one conventions to the ones used in another one. In general, those relations will not be free of kinematical poles, because they are obtained by solving sets of coupled linear equation, which by Cramer's rule are found as ratios of determinants.

\subsubsection{A Novel Projection Method}

The projection methods we discussed in the previous sub-section share the occurrence of single and double poles from the beginning, which must be removed to obtain a formulation of the Compton tensor free of kinematical singularities. Here we propose a method that is free of poles ab inito so that no regularisation is necessary. It will serve as the back bone of the Compton tensor. To this back bone, pairs of momenta are fixed by contraction, like the base pairs in DNA. So we define

$$
d^{\mu \nu \alpha \beta}=g^{\mu \nu} g^{\alpha \beta}-g^{\mu \beta} g^{\nu \alpha} .
$$

We note that $d^{\mu \nu \alpha \beta}$ is symmetric under the simultaneous interchange $\mu \leftrightarrow \nu, \alpha \leftrightarrow \beta$ and changes sign by the interchanges $\mu \leftrightarrow \alpha$, and $\nu \leftrightarrow \beta$. Using this back bone we construct pieces of "DNA" by contracting it with the three basis four vectors. With an obvious notation we write them as follows:

$$
\begin{aligned}
G^{\mu \nu}\left(q^{\prime} q\right) & =q_{\alpha}^{\prime} d^{\mu \nu \alpha \beta} q_{\beta}=q^{\prime} \cdot q g^{\mu \nu}-q^{\mu} q^{\prime \nu}, \\
G^{\mu \nu}(q q) & =q_{\alpha} d^{\mu \nu \alpha \beta} q_{\beta}=q^{2} g^{\mu \nu}-q^{\mu} q^{\nu}, \\
G^{\mu \nu}\left(q^{\prime} q^{\prime}\right) & =q_{\alpha}^{\prime} d^{\mu \nu \alpha \beta} q_{\beta}^{\prime}=q^{\prime 2} g^{\mu \nu}-q^{\prime \mu} q^{\prime \nu}, \\
G^{\mu \nu}(\bar{P} q) & =\bar{P}_{\alpha} d^{\mu \nu \alpha \beta} q_{\beta}=\bar{P} \cdot q g^{\mu \nu}-q^{\mu} \bar{P}^{\nu}, \\
G^{\mu \nu}\left(q^{\prime} \bar{P}\right) & =q_{\alpha}^{\prime} d^{\mu \nu \alpha \beta} \bar{P}_{\beta}=\bar{P} \cdot q^{\prime} g^{\mu \nu}-\bar{P}^{\mu} q^{\prime \nu} .
\end{aligned}
$$

The first tensor is identical with $q^{\prime} \cdot q$ times Tarrach's projector, the second and the third ones are multiples of the projectors used by Perrottet. The last two are novel. Including $\bar{P}$ in the set of building blocks of projectors, more freedom in the construction of the transverse tensor is created. These five tensors have vanishing contractions with $q_{\mu}^{\prime}$ and $q_{\nu}$ and are free of kinematical singularities ab initio. The latter property obviates the necessity of the Tarrach construction to remove the single and double poles.

Given these building blocks the transverse tensor $\tilde{T}_{D N A}^{\mu \nu}$ can be written as follows

$$
\begin{aligned}
\tilde{T}_{\mathrm{DNA}}^{\mu \nu}:=\sum_{i=1}^{5} \mathcal{S}_{i} \tilde{T}_{\mathrm{DNA}}^{(i) \mu \nu}= & \mathcal{S}_{1} G^{\mu \nu}\left(q^{\prime} q\right) \\
& +\mathcal{S}_{2} G^{\mu \lambda}\left(q^{\prime} q^{\prime}\right) G_{\lambda}{ }^{\nu}(q q) \\
& +\mathcal{S}_{3} G^{\mu \lambda}\left(q^{\prime} \bar{P}\right) G_{\lambda}{ }^{\nu}(\bar{P} q) \\
& +\mathcal{S}_{4}\left[G^{\mu \lambda}\left(q^{\prime} \bar{P}\right) G_{\lambda}{ }^{\nu}(q q)+G^{\mu \lambda}\left(q^{\prime} q^{\prime}\right) G_{\lambda}{ }^{\nu}(\bar{P} q)\right] \\
& +\mathcal{S}_{5} G^{\mu \lambda}\left(q^{\prime} q^{\prime}\right) \bar{P}_{\lambda} \bar{P}_{\lambda^{\prime}} G^{\lambda \nu}(q q) .
\end{aligned}
$$

By direct computation one may check that the DNA representation is simply related to Metz's as given in Eq. (39):

$$
\tilde{T}_{\mathrm{DNA}}^{(1)}=-M_{1}, \quad \tilde{T}_{\mathrm{DNA}}^{(2)}=M_{3}, \quad \tilde{T}_{\mathrm{DNA}}^{(3)}=-M_{2}, \quad \tilde{T}_{\mathrm{DNA}}^{(4)}=M_{4}, \quad \tilde{T}_{\mathrm{DNA}}^{(5)}=M_{19} .
$$

The tensor $M_{19}$ does not fit immediately in the Bardeen-Tung construction, but was introduced in Ref. [16] as $T_{19} \equiv M_{19} / q^{\prime} \cdot q$ together with two other ones that can only occur for spin- $1 / 2$ targets, in order to create more freedom to construct the Compton tensor. Metz used this tensor to replace another one in his original transverse basis. We shall not discuss this matter in more detail, but just note that in the DNA construction this tensor occurs quite naturally.

A final remark is in order here. In the literature sometimes one sees representations of the Compton tensor that are not manifestly transverse. In those cases use has been made of the equations of motion for the wave 
functions of the external particles, hadrons and photons. Such a representation has the disadvantage that because terms have been omitted, a check of the original equation is not possible anymore. For an illustration we take a look at the tree-level tensor $T_{\text {tree }}^{\mu \nu}$. To calculate the amplitudes, we must use Eq. (12). Using the definition $\bar{P}=p^{\prime}+p$, four-momentum conservation $p^{\prime}+q^{\prime}=p+q$, and the property of the polarisation vectors $\epsilon_{\mu}^{*}\left(q^{\prime} ; h^{\prime}\right) q^{\prime \mu}=0=q^{\nu} \epsilon_{v}(q ; h)$ we can reduce $T_{\text {tree }}^{\mu \nu}$ to a simpler form, namely

$$
T_{\mathrm{eff}}^{\mu \nu}=-2 g^{\mu \nu}+4 \frac{p^{\prime \mu} p^{\nu}}{s-M^{2}}+4 \frac{p^{\mu} p^{\prime \nu}}{u-M^{2}},
$$

which has the same contractions with the polarisation vectors but is, however, not transverse.

\section{Spin Filter}

The form of the Compton tensor used by Metz or the one constructed using the DNA approach produces for any physical situation, i.e. $q^{\prime 2}=0, q^{\prime} \cdot q=0, q^{2}=0$ and $\bar{P} \cdot q^{\prime}=\bar{P} \cdot q=0$, five independent components and thus depends on five CFFs. Nevertheless, the amplitudes calculated by contracting the tensor with the photon polarisation vectors may depend on less than five CFFs. We may say that they form a kind of spin filter [17]. The reason why this works is easily understood in the Tarrach representation Eq. (35). Consider the transverse four vector $\tilde{q}_{\mathrm{L}}^{\prime \mu}$ :

$$
\tilde{q}_{\mathrm{L}}^{\prime \mu}=q^{\prime \mu}-\frac{q^{\prime 2}}{q^{\prime} \cdot q} q^{\mu}
$$

This four vector is a left-hand factor in $\tilde{T}_{3}^{\mu \nu}$ and $\tilde{T}_{4}^{\mu \nu}$. While $\epsilon_{\mu}\left(q^{\prime} ; h^{\prime}\right)$ will annihilate $q^{\prime \mu}$ these two components of the transverse tensor will survive when $q^{\prime 2} \neq 0$, but in the case that the photon in the final state is real, the second part of $\tilde{q}_{\mathrm{L}}^{\prime \mu}$ vanishes, which effectively filters out the CFFs $\mathcal{H}_{3}$ and $\mathcal{H}_{4}$ from the amplitudes. A similar situation occurs when the incoming photon is real. Then $\mathcal{H}_{2}$ and $\mathcal{H}_{4}$ are filtered out. Finally, in real Compton scattering, only two CFFs are visible, namely $\mathcal{H}_{0}$ and $\mathcal{H}_{1}$.

This filter principle is reflected in the number of effective degrees of freedom in Compton scattering. In the case that both photons are virtual, there exist nine combinations of the photon helicities in the initial and final states, $h^{\prime}, h \in\{1,0,-1\}$. Parity conservation gives for the amplitudes $A\left(-h^{\prime},-h\right)=(-1)^{h^{\prime}-h} A\left(h^{\prime}, h\right)$ from which it follows that there are five independent amplitudes, for instance

$$
A(1,1), A(1,0), A(1,-1), A(0,1) \text {, and } A(0,0) \text {. }
$$

In the case that $q^{\prime 2}=0$, the number of helicities in the final state is reduced to two: $h^{\prime}=1,-1$. Then the number of independent amplitudes is also reduced to three, for instance $A(1,1), A(1,0)$, and $A(1,-1)$, and for real Compton scattering this number is again reduced, namely to two: $A(1,1)$ and $A(1,-1)$. This counting reflects the number of visible CFFs in these various kinematical regimes. This circumstance that the number of independent amplitudes is identical to the number of visible CFFs, provides a reason to believe that one can invert Eq. (12) to find the CFFs in terms of the helicity amplitudes. Such an extraction is indeed possible, but it is known [18] that this procedure can be very sensitive to uncertainties in the amplitudes. As in actual practice the amplitudes must be determined from experimental data, one should not expect that such an inversion will give accurate values of all visible CFFs.

\section{Epilogue}

It is clear now that there is a multitude of forms of the Compton tensor even for a scalar particle. How to choose a particular one is partly a matter of taste, but is sometimes motivated by arguments inspired by ideas about the physical structure of the target hadron. In deeply-virtual Compton scattering off the proton the partonic structure of the target is the main focus. Then the operator-product expansion gives a hint as to the relative importance of the GPDs. This motivation has guided the choice of the form of the Compton tensor in the literature, see for instance Ref. [19-23].

This review being devoted to the case of Compton scattering on a scalar target, does not touch the subtleties of including $\gamma^{\mu}$ as a fourth basis vector in the construction of the Compton tensor. The works of Perrottet, 
Tarach, and Metz, among others, show the results of using the projector method to construct $T^{\mu v}$ for a spin-1/2 target. As they sometimes utilise the equations of motion of the target wave function, the Dirac equation, the transversality of the published tensors is not manifest. This point was touched also in Sect. 3, where six forms of the current operator $J^{\mu}$ were shown, some of which being manifestly transverse, but others can only be proved to be transverse after using the Dirac equation.

Possibly, the direct method and the DNA approach could be made to work for spin-1/2 targets too. This point is to our knowledge open for closer investigation.

Acknowledgements This work was supported in part by the DOE Contract No. DE-FG02-03ER41260.

Open Access This article is distributed under the terms of the Creative Commons Attribution 4.0 International License (http:// creativecommons.org/licenses/by/4.0/), which permits unrestricted use, distribution, and reproduction in any medium, provided you give appropriate credit to the original author(s) and the source, provide a link to the Creative Commons license, and indicate if changes were made.

\section{References}

1. J.D. Jackson, Classical Electrodynamics, 3rd edn. (Wiley, New York, 1998)

2. S. Weinberg, The quantum theory of fields (Cambridge University Press, Cambridge, 1995)

3. J.D. Bjorken, S.D. Drell, Relativistic Quantum Mechanics (McGraw-Hill, New York, 1964)

4. F.J. Ernst, R.G. Sachs, K.C. Wali, Electromagnetic form factors of the nucleon. Phys. Rev. 119, 1105 (1960)

5. R.G. Sachs, High-energy behaviour of nucleon electromagnetic form factors. Phys. Rev. 126, 2256 (1962)

6. W. Gordon, Der Strom der Diracschen Elektronentheorie. Z. Phys. 50, 630 (1928)

7. C.-R. Ji, B.L.G. Bakker, H.-M. Choi, A. Suzuki, Ideas of four-fermion operators in electromagnetic form factor calculations. Phys. Rev. D 87, 093004 (2013)

8. A.H. Compton, A quantum theory of the scattering of X-ray by light. Phys. Rev. 21, 483 (1923)

9. P. Debye, Zerstreuung von Röntgenstrahlen und quantentheorie. Phys. Zeitschr. 24, 161 (1923)

10. A. Pais, Subtle is the Lord (The Science and Life of Albert Einstein) (Oxford University Press, New York, 1982)

11. A.V. Belitsky, D. Müller, Refined analysis of photon leptoproduction off a spinless target. Phys. Rev. D 79, 014017 (2009)

12. K. Kumerički, D. Müller, Deeply virtual Compton scattering ar small $x_{\mathrm{B}}$ and the access to the GPD $H$. Nucl. Phys. B 841, $1(2010)$

13. M. Perrottet, Invariant amplitudes for Compton scattering of off-shell photons on polarised nucleons. Lett. Nouvo Cim. 7, 915 (1973)

14. R. Tarrach, Invariant amplitudes for virtual compton scattering off polarized nucleons free from kinematic singularities, zeros and constraints. Nuovo Cim 28 A, 409 (1975)

15. Metz, M.: Virtuelle Comptonstreuung und die Polarisierbarkeiten des Nukleons (in German), PhD thesis, Universität Mainz, (1997)

16. W.A. Bardeen, W.-K. Tung, Invariant amplitudes for photon processes. Phys. Rev. 173, 1423 (1968)

17. B.L.G. Bakker, C.-R. Ji, Spin filter indeeply virtual Compton scattering amplitudes. Phys. Rev. D 83, 091502 (2011)

18. B.L.G. Bakker, C.-R. Ji, Extraction of compton form factors in scalar QED. Few-Body Syst 56, 275 (2015)

19. X.D. Ji, Gauge invariant decomposition of nucleon spin. Phys. Rev. Lett 78, 610 (1997)

20. X.D. Ji, Gauge invariant decomposition of nucleon spin. Phys. Rev. D 55, 7114 (1997)

21. A.V. Radyushkin, Scaling limit of deeply virtual Compton scattering. Phys. Lett. B 380, 417 (1996)

22. A.V. Radyushkin, Nonforward parton distributions. Phys. Rev. D 56, 5524 (1997)

23. D. Mueller, D. Robaschik, B. Geyer, F.M. Dittes, J. Horejsi, Wave functions, evolution equations and evolution kernels from light-ray operators of QCD. Fortsch. Phys. 42, 101 (1994). [arXiv:hep-ph/9812448] 